\title{
Article \\ ON THE LASER DOPPLER VELOCIMETRY FOR SURFACE ROTATION MEASUREMENTS
}

\author{
Dawid Kucharski * (1), Mateusz Musiałowski \\ Division of Metrology and Measurement Systems; Institute of Mechanical Technology; Faculty of Mechanical \\ Engineering; Poznan University of Technology; Jana Pawla II 24 Street; 60-965 Poznan; Poland. \\ * $\quad$ dawid.kucharski@put.poznan.pl; Tel.: +48 616653269
}

\begin{abstract}
The article aims to present the possible use of the simple modified optical training kit as a low-cost, simple setup for high precision surface speed measurements. A measurement capability evaluation of the optical Doppler kit used for velocimetry of a fast-rotating reflecting surface is presented. To get high repeatability measurements, we modified a fibre optic interferometry training kit. By using signal processing and statistics a precision under repeatability conditions of measurements was evaluated. Expressed by the standard deviations $\left(3 \sigma^{\prime} \mathrm{s}\right)$ the surface velocity measurements precision below $0.2 \frac{\mathrm{m}}{\mathrm{s}}$ is shown. The $C_{g}$ 's capability indices were also evaluated. We postulate, the electric circuit stability of the measurement system power supply is essential for a signal noise reducing process for a wide range of metrology systems. It is crucial for precision measurements.
\end{abstract}

Keywords: Doppler effect, optical measurements, precision, rotation velocimetry, system capability, repeatability, surface metrology

\section{Introduction}

The light-matter interaction can turn out as a complex network of the electromagnetic field. It can be physically and mathematically described nowadays quite widely and precisely. Because of the redefined in 2019 the SI units, based on the speed of light in a vacuum (c) [1,2], this beautiful part of nature now is commonly used in metrology for high precision, traceable measurements [3]. The light is already used in the manufacturing metrology for fast surface measurements in a various scale [4-7]. Except for high-accuracy interferometry [8], the velocity measurements of these fast-moving surfaces can also be determined precisely by using well known optical Doppler effect (non-relativistic) [9-14], as well as surface asperities. It can be considered even parallel in one measurement system, where surface topography and displacement are measured together, with comparable precision [15]. It is possible e.g. with interferometric surface texture measurement technique [16].

The Doppler radar technique has a wide application from technical science $[17,18]$ to life science as well [19]. By using the Doppler effect principle, the vital signals are also measured in bio-radar systems for motion detection applications [20]. A groundbreaking in science was taking into account the Doppler effect by Albert Einstein in the relativity theory with time dilatation effect in 1905 [21]. So, the Doppler shift principle was, and is a decisive part of the wave theory, modern physics and modern technical science.

An industrial laser Doppler velocimetry method has been developed for accurately measuring the velocity and length of moving surfaces already in 1983 [22]. Simultaneous measurement of the velocity and the displacement of the surface by a laser Doppler velocimeter is also well known [23]. The systems also have been miniaturized [24], but even nowadays, these systems are rather advanced, innovative and costly. In the paper, a simple, modified fibre optical training kit $[25,26]$, for high precision velocimetry of a fast-rotating reflecting surface, is proposed. By using the isolation transformers, the laboratory electrical grid from the building grid noise was separated. A simple, stabilised, power supply for DC engine was prepared. We proved, electric noise reduction and stable power supply 
are essential for frequency measurements. They are especially important for high precision optical velocimetry using the Doppler kit, under repeatability conditions of measurements. The setup can be adapted as a non-lab, low-price measurement system, to measure the velocity of the moving surface up to $30 \frac{\mathrm{m}}{\mathrm{s}}$. Further investigations with various surfaces will begin presently.

We aim to construct an optical measurement system based on lasers, for surface speed displacement and surface topography measurements with both nanometric precision, to be as a low-cost, fast, simple system, not necessarily high innovative, without an expensive, advanced optics. As the first part of the system, we propose to use our modified Doppler training kit. A second part might be based on scatterometric [27] or interferometric systems [16]. In the paper, the precision of the Doppler system measurements was evaluated by statistics.

\subsection{The Doppler shift}

Consider a source ( $\mathrm{S}$ in fig. 1) emits an electromagnetic wave towards a reflecting surface (O in fig. 1). The reflected wave seems to come from $S^{\prime}$. If surface moves at speed $v<<c, S^{\prime}$ goes at speed $2 v$. It is related to the double change of the optical path length, caused by the surface displacement. The wave frequency detected by the receiver ( $R$ in fig. 1 ) is shifted. The effect is well known as the non-relativistic Doppler shift [28]. The laser speed gun works in this way.

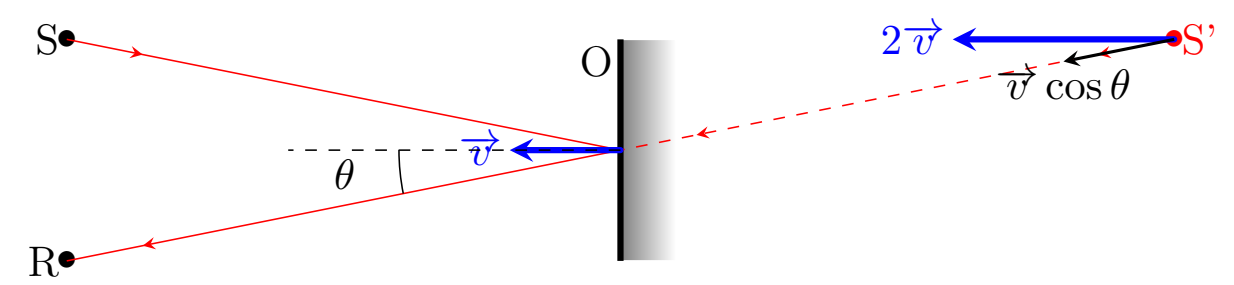

Figure 1. The Doppler shift principle. S - wave source, R - receiver, $S^{\prime}$ - virtual wave source, $\mathrm{O}$ object (surface). Based on [29]

We used the optical setup to determine the tangential speed of the rotating disc by using the Doppler principle. The speed vectors relation scheme is shown in fig. 2 . The vectors are oriented in a 3D space.

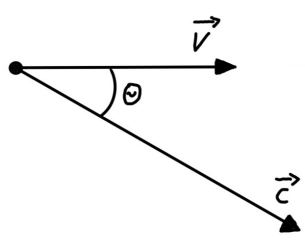

A

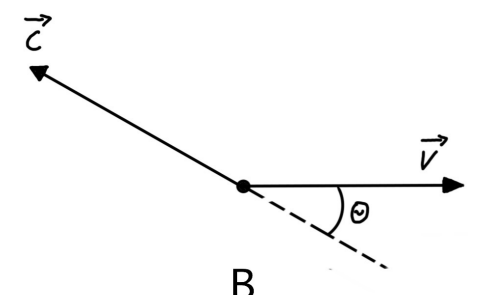

B

Figure 2. Angle relation between the speed of the disc $\vec{v}$ and the speed of light $\vec{c}$ : A-wave reception by the disc surface, B-diffused wave emission. Based on [25]

Single-mode laser light $(\lambda=1550 \mathrm{~nm})^{\mathrm{a}}$ has been reflected from a disc covered by retrodiffusing layer. The frequency of the incindent light is $f_{0}$ (see eq. 1) [25].

$$
f_{0}=\frac{c}{\lambda}=\frac{3 \times 10^{8}}{1550 \times 10^{-9}} \simeq 193.6[\mathrm{THz}]
$$

where:

a $\quad \Delta \lambda< \pm 1 \mathrm{~nm}[25]$ 
$c$ - the speed of light,

$\lambda$ - the wavelength $(1550 \mathrm{~nm})$.

When the beam hits the rotating disc, the frequency shifts twice: at the wave reception by the disc, and the diffused wave emission by the same disc. For the same direction of the disc rotation as the direction of the light, the frequency detected by the disc is (see eq. 2 and fig. 2):

$$
f_{1}=f_{0} \cdot\left(1-\frac{v \cdot \cos \theta}{c}\right)
$$

where:

$f_{0}$ - frequency of the incident light,

$f_{1}$ - received frequency by the rotating disc,

$v$ - speed of the disc,

$\theta-\Varangle(\vec{v}, \vec{c})$.

The reemitted frequency (went to collimator) $f_{2}$ is described by the eq. 3 .

$$
f_{2}=\frac{f_{1}}{1+\frac{v \cos \theta}{c}} .
$$

Because $v<<c$, we obtain from eq. 3, eq. 4:

$$
f_{2} \approx f_{0} \cdot\left(1-\frac{2 v}{c} \cos \theta\right) .
$$

Hence the Doppler shift (eq. 5):

$$
|\Delta f|=\left|f_{2}-f_{0}\right| \approx \frac{2 f_{0} v \cos \theta}{c}
$$

By detecting the Doppler shift $|\Delta f|$, with determined $\theta-\Varangle(\vec{v}, \vec{c})$, the speed of the disc can be estimated as follows (eq. 6):

$$
v=\frac{|\Delta f| \cdot c}{2 f_{0} \cos \theta}\left[\frac{m}{s}\right] .
$$

\section{Setup}

The optical layout of the setup is shown in fig. 3.

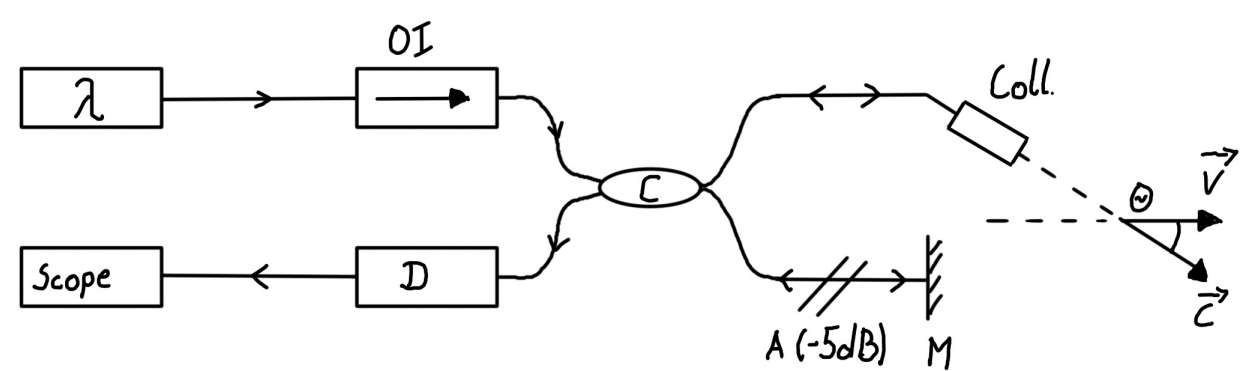

Figure 3. Optical layout: $\lambda$ - semiconductor laser $(P=1 \mathrm{~mW}$, wavelength - $1550 \mathrm{~nm})$, Scope oscilloscope (Rigol DS1052E), OI - optical isolator, D - detector, C - fibre coupler, A - attenuator (-5 dB), M - mirror, Coll. - optical collimator. Based on [25]

The setup was laying on the granite isolation table (5 in fig. 4) for mechanical and acoustic noise reduction. The fibre interferometry and Doppler kit (see fig. 4) was additionally separated by an aluminium plate with four rubber feet (Sorbothane feet, $\varnothing 45 \mathrm{~mm}$ ). To keep a constant speed of the rotation (1600 rpm), surface to be measured ( $\varnothing 75 \mathrm{~mm}$ reflecting disc, see fig. 5) was mounted on a DC engine supplied by the self-built stabilised power supply $\left(U=3 \mathrm{~V}, I_{\max }=850 \mathrm{~mA}\right.$ ) (see fig. 6). 


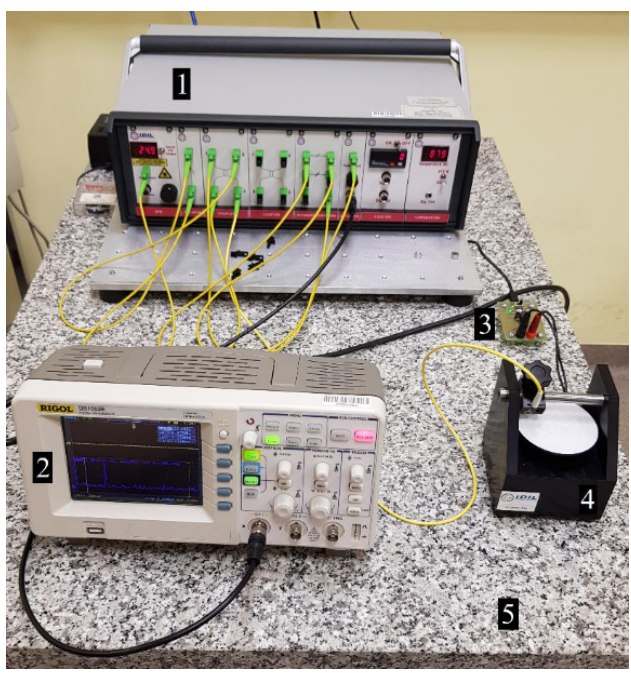

Figure 4. The fibre optic interferometry and Doppler kit: 1 - semiconductor laser with optics, 2 - scope (Rigol DS1052E), 3 - power supply (see fig. 6), 4 - $\varnothing 75$ $\mathrm{mm}$ optical disc with reflecting surface, mounted on the DC engine (nominal voltage - $3 \mathrm{~V}$, nominal rotation speed $1600 \mathrm{rpm}$ ), 5 - granite table (vibration isolation) $[25,26]$

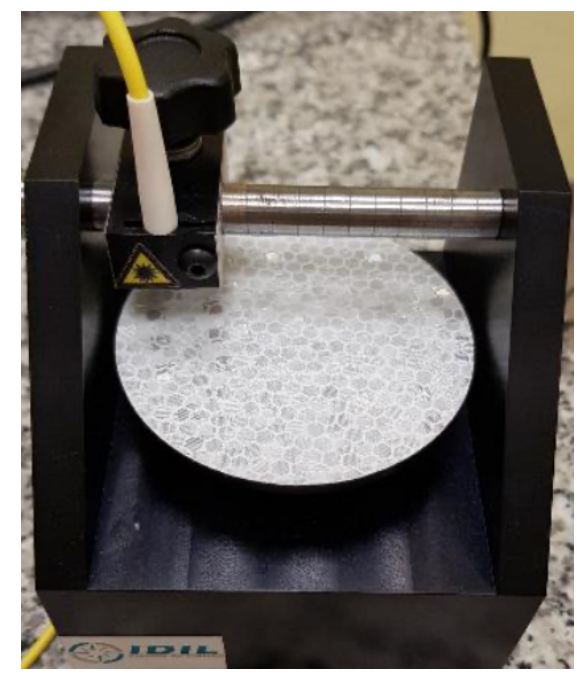

Figure 5. Optical disc $(\varnothing 75 \mathrm{~mm})$ with reflecting surface, mounted on the DC engine (nominal voltage - $3 \mathrm{~V}$, nominal rotation speed - $1600 \mathrm{rpm}$ ) $[25,26]$

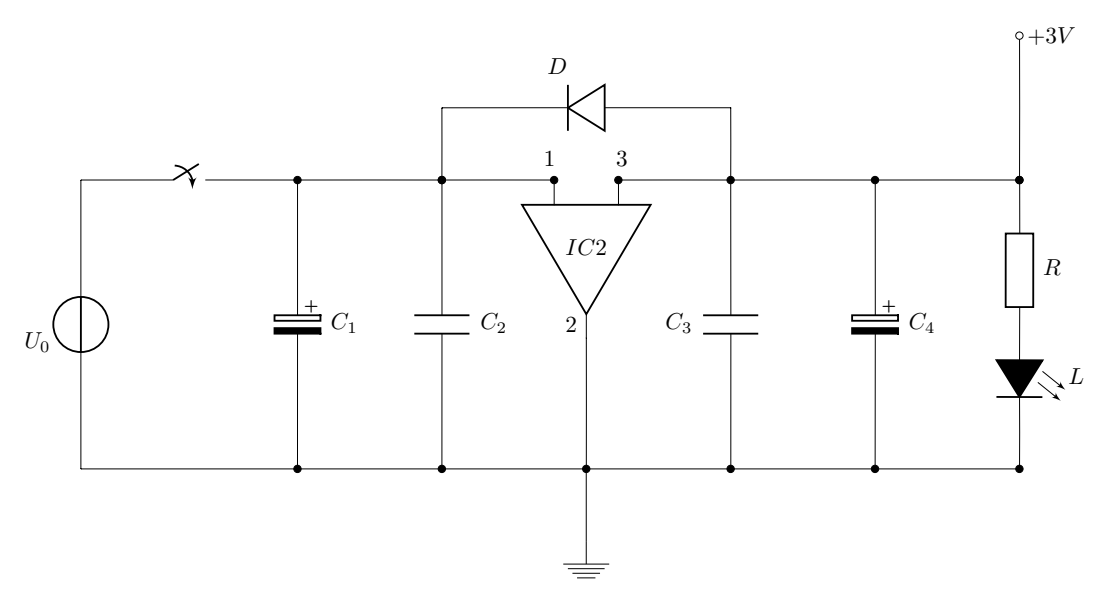

Figure 6. Power supply scheme ( 3 in fig. 4): IC2 - linear voltage stabiliser LM1117T (3.3 V), $C_{1-4}$ capacitors for high-frequency noise filtering $\left(C_{1,4}=470 \mu \mathrm{F}, C_{2,3}=100 \mathrm{nF}\right), D$ - diode (1N4004) to protect the stabiliser against too high backward voltage, $R$ - resistor $(1 \mathrm{k} \Omega), L$ - LED

A typical power source was used in the experiment, from the electrical grid of the building $(V=230 \mathrm{~V}, 50 \mathrm{~Hz})$. To reduce the electric noise from many types of equipment in a whole building, the electrical grid of the laboratory was separated by the transformers $(12 \mathrm{kVA} ; 0.4 / 0.4 \mathrm{kV} ; R \approx 4 \mathrm{M} \Omega$ ). In this way, repeatable electric conditions for the experiment, not depending on the activities in the building, were prepared. The data were collected by using a scope (2 in fig. 4). Optical elements of the system were connected using optical fibres ${ }^{b}$ and fibre APC connectors ${ }^{c}$, as mentioned in the fig. 3.

b Gold mirrored fibre ends

c Patchcords E2000/APC Diamond connectors; APC - Angled Physical Contact 
The laser beam $(P=1 \mathrm{~mW}, \lambda=1550 \mathrm{~nm})$ is sent through the optical isolator (OI in fig. 3 ), to avoid damage of the laser resonator. Then, the fibre coupler ( $\mathbf{C}$ in fig. 3 ) is split the beam into the two channels of the setup. One with the mirror as the reference ( $\mathbf{M}$ in fig. 3) and the second as a measurement one with fibre collimator (Coll. in fig. 3). The mirror improves the carrier frequency.

To reduce the power of the reference beam and makes equal two beams mixed into the one signal detected by the photodiode ( $\mathbf{D}$ in fig. 3$)^{\mathrm{d}}$, the $-5 \mathrm{~dB}$ optical attenuator (A in fig. 3 ) is placed in the reference channel, just before the mirror. The attenuator also avoids a photodiode saturation.

\section{Methods}

\subsection{The measurement procedure}

The scheme of the measurement procedure is shown in fig. 7. Before the measurements, the parameters are set: current of the laser diode $(I=25 \mathrm{~mA}$, kept always the same), photodiode offset $\left(V_{\text {offset }}=1-4 \mathrm{~V}\right)$, reflection angle $\left(\theta=88.5,78.75,67.50^{\circ}\right)$ and the measurement place on the disc (radius $R=20,25,30 \mathrm{~mm}$ ). Next, the DC motor is switched on, to achieve, after c.a. 30 seconds, a constant speed of the disc rotation. The signal is detected by the scope and is stored on the external USB stick for further evaluation. This procedure was repeated 30 times for the setup capability evaluation (see fig. 7).

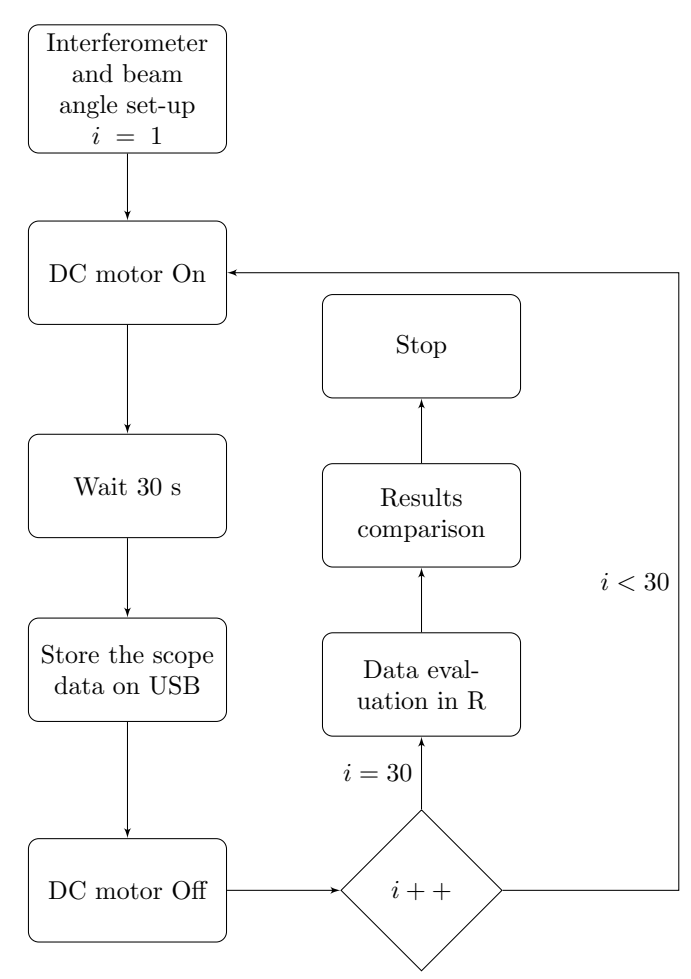

Figure 7. The measurement procedure scheme

The measurement procedure shown in fig. 7 corresponds to one set of parameters. Consider the parameters set as: $I=25 \mathrm{~mA}, V_{\text {offset }}=1 \mathrm{~V}, \theta=88.5^{\circ}, R=30$, and the disc started to rotate with constant speed $1600 \mathrm{rpm}$. After c.a. $30 \mathrm{~s}$, the signal from the scope is stored on the USB stick. Next, the DC motor is switched off to stop the disc. For a measurement repeatability investigation, the above procedure was repeated 30 times. The data from the scope as the ${ }^{*}$ dat files were imported in $R$

d InGaS photodiode $(\mathrm{BW}>10 \mathrm{MHz})$ 
programming language. The signal processing and statistics were evaluated by using the additional $\mathrm{R}$ package [30,31].

\subsection{Signal processing}

For Doppler shift evaluation, the fast Fourier transform was used. In fig. 8 example of the photodiode signal, registrered by the scope, is depicted. The sampling interval was always $10^{-8} \mathrm{~s}(8192$ data points). The signal transform to the frequency domain is presented in fig. 9. The DC component clearly to be seen, comes from the photodiode offset $\left(V_{o f f s e t}=1 \mathrm{~V}\right)$, and only negligibly affects the Doppler peak analysis.

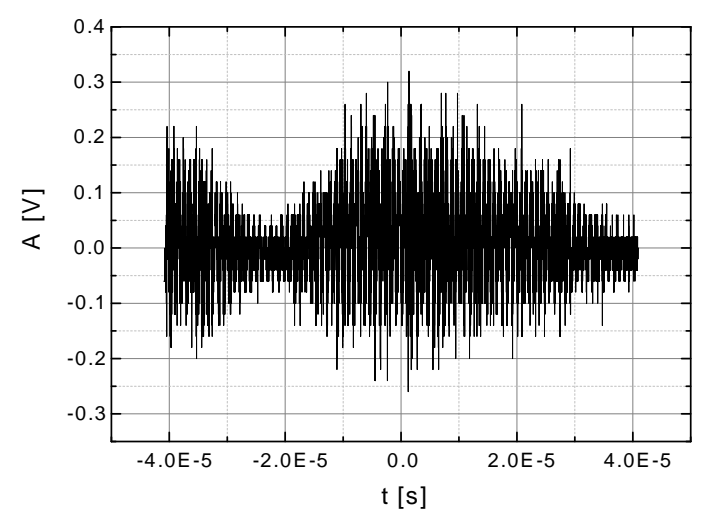

Figure 8. Example of the photodiode signal detected by the scope during the measurments

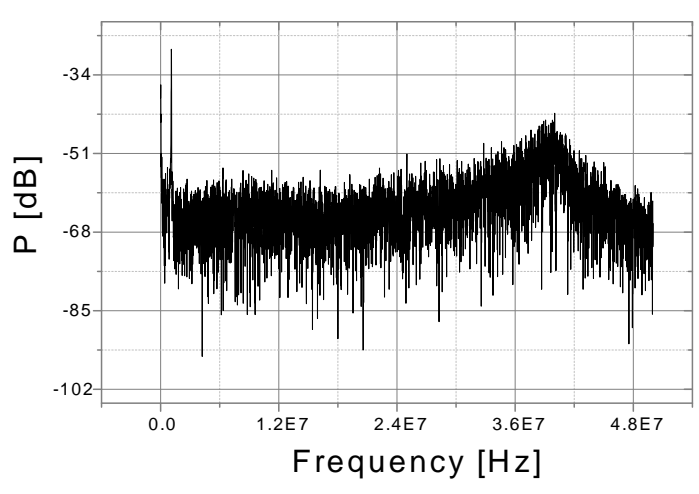

Figure 9. Example of the fast Fourier transform of the signal (in log scale) from fig. 8. $P$ is the power spectrum of the signal. Sampling interval was $10^{-8}$

Before Doppler peak evaluation, a low-pass filter was used with cutoff frequency $f_{c}=4.2 \times 10^{-6}$ (see fig. 10 and 11 ).

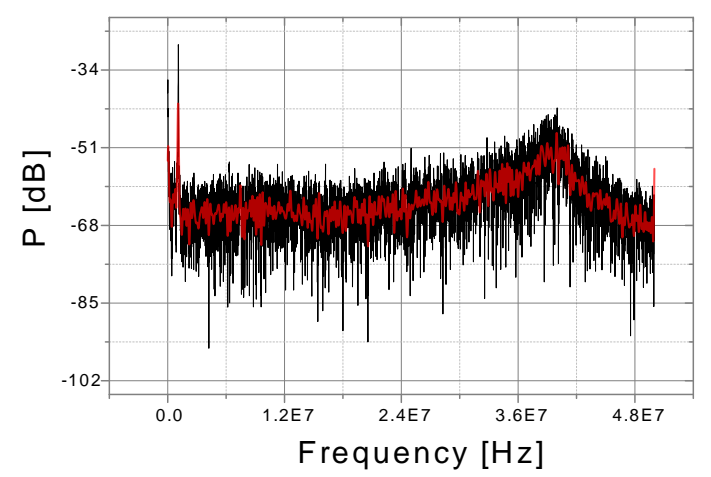

Figure 10. Power spectrum of the signal with low-pass filter (red line) (cutoff frequency $f_{c}=4.2 \times 10^{-6}$ )

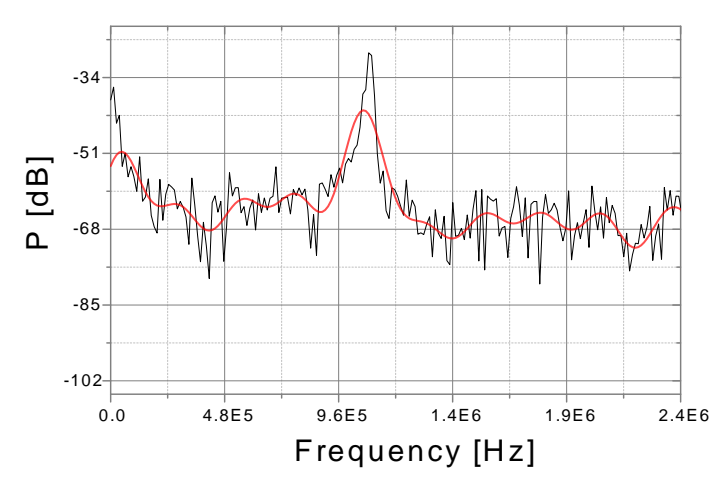

Figure 11. The power spectrum of the signal with a low-pass filter (red line). The graph is scaled for a close-range around the Doppler peak

Next steps are, to fit the filtered spectrum by using the Gaussian curve, and Doppler peak detection. The Doppler peak is found by using a 2nd derivative of the Gaussian curve (see fig. 12). 


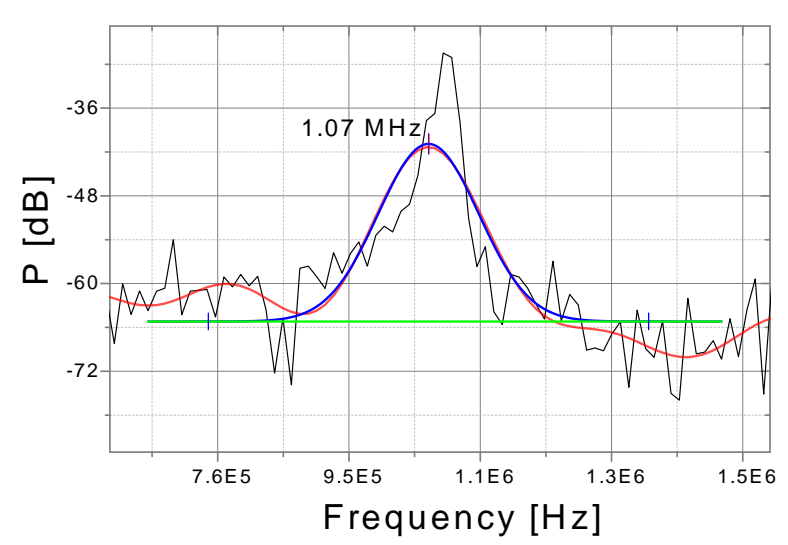

Figure 12. The power spectrum of the signal with a low-pass filter (red line) and the Gaussian fit (blue line - 66 data points). The baseline of the Gaussian curve (as the minimum) is depicted by the green line

In fig. 9 a high frequency noise is presented. The data were stored without using the separation transformers in the laboratory. The high frequency noise was reduced in the final setup with transformers (see fig. 13). The increased DC compomnent comes from the photodiode offset $V_{\text {offset }}=4 \mathrm{~V}$, used in the final experiment.

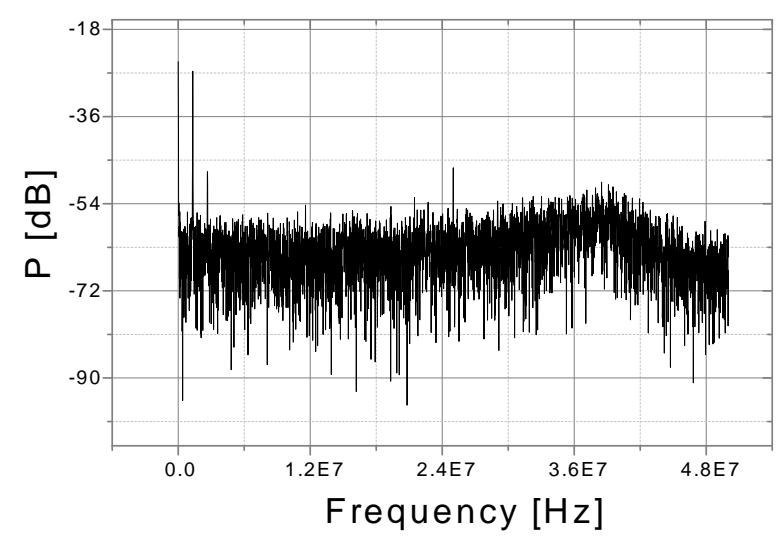

Figure 13. Example of the fast Fourier transform of the signal (in log scale), detected after the separation transformers were used in the laboratory. $P$ is the power spectrum of the signal. The DC component increased in a comparison with the DC from fig. 9, due to the higher photodiode offset $\left(V_{\text {off } f \text { set }}=4 \mathrm{~V}\right)$

\subsection{Disc speed evaluation}

By using the Gaussian fit, the Doppler peak (see e.g. fig. 12) can be determined. For known frequency of the Doppler shift, the disc speed can be calculated from the eq. 6. Because of a lack of measurement standard, for measurement system capability evaluation, the tolerance limits from the speed fluctuations $(v)$ due to the power voltage $\left(U_{z}\right)$ were estimated. The constant $(k)$ of the motor velocity is described by the eq. 7 .

Table 1. The DC motor parameters

\begin{tabular}{cc}
\hline Rotation speed $[\mathrm{rpm}]$ & Power voltage $[\mathrm{V}]$ \\
\hline$\omega_{s}=1600$ & $U_{z}=3.3$ \\
$k=\frac{\omega_{s}}{U_{z}}=\frac{1600}{3.3} \approx 484.85\left[\frac{\mathrm{rpm}}{\mathrm{V}}\right]$,
\end{tabular}

where: $\omega_{s}$ and $U_{z}$ values are mentioned in tab. 1 .

Measured instability of the power voltage was (see eq. 8): 


$$
\Delta U_{z}= \pm 0.05[V] .
$$

From eq. 7 and 8 a maximum error of the motor rotation speed can be described as (eq. 9):

$$
\Delta \omega_{s}=\left|\frac{\partial \omega_{s}}{\partial U_{z}}\right| \cdot \Delta U_{z}=k \cdot \Delta U_{z}=484.85 \cdot 0.05 \approx 24.24[\mathrm{rpm}],
$$

and the angular speed, finally as (eq.10):

$$
\omega \approx 167.55 \pm 2.54\left[\frac{\mathrm{rad}}{\mathrm{s}}\right] .
$$

The linear speed $(v)$ for $R=0.03[\mathrm{~m}]$ is described by the eq. 11. In fig. 14 the linear relation from eq. 11 is presented. It was proved experimentally (see section 4, fig. 20). The Doppler shift varies as the cos function (see eq. 6 and fig. 15).

$$
v=\omega \cdot R \approx 5.02 \pm 0.08\left[\frac{m}{s}\right]
$$

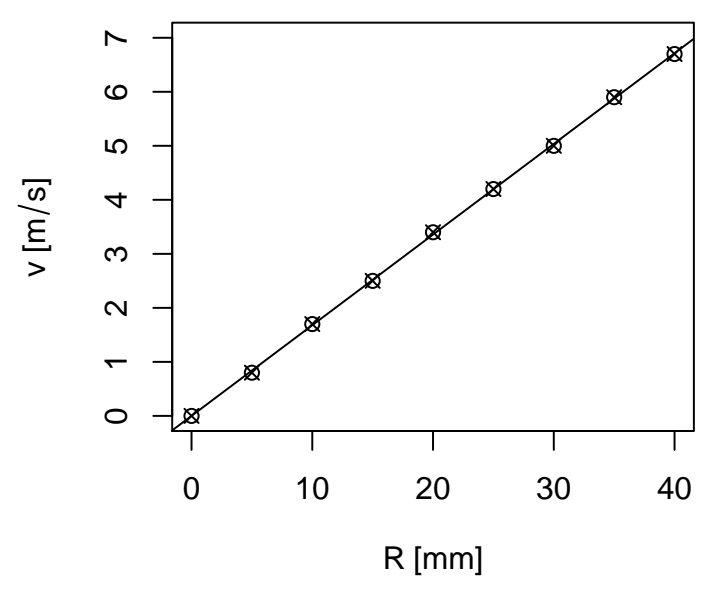

Figure 14. Linear speed $(v)$ versus disc radius $(R)$ graph. Related to the eq. 11

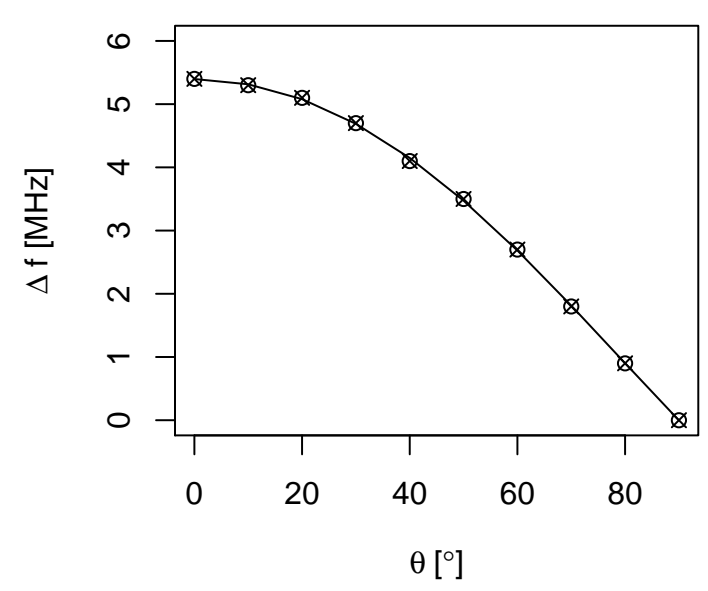

Figure 15. Doppler shift $(\Delta f)$ versus angle $(\theta)$ graph. Related to the eq. 6

From theoretical linear speed (eq. 11) of the disc in radius $R=0.03[\mathrm{~m}]$, upper tolerance limit (UTL), lower tolerance limit $(L T L)$ and the tolerance range $T_{R}$ (see eq. 12) were defined.

$$
\begin{aligned}
U T L=v+\Delta v & =5.10\left[\frac{\mathrm{m}}{\mathrm{s}}\right], \\
L T L=v-\Delta v & =4.94\left[\frac{\mathrm{m}}{\mathrm{s}}\right], \\
T_{R=30}=|U T L-L T L| & =0.16\left[\frac{\mathrm{m}}{\mathrm{s}}\right] .
\end{aligned}
$$

In the same way, linear speed tolerances for different points (radiuses $R$ ) on the disc (see eq. 13 were calculated.

$$
\begin{aligned}
T_{R=25} & =0.12\left[\frac{m}{s}\right], \\
T_{R=20} & =0.10\left[\frac{m}{s}\right] .
\end{aligned}
$$

For the velocimetry system capability evaluation, well known in manufacturing metrology, the standard Measurement Systems Analysis (MSA) method [32,33] is proposed. The method is widely 
used [34,35]. In our case, a standard deviation parameter $(3 \sigma)$ and the $C_{g}$ metric (see eq. 14) were used for the precision estimation, under repeatability conditions of measurement. Measurements were repeated 30 times per series (total 18 series). To check the normal distribution of the data the Shapiro-Wilk test [36] was used. We used the open-source programming language $R$ for statistical computing [31,37,38].

$$
C_{g}=\frac{T}{6 \sigma}
$$

where:

$T$ - speed tolerance $\left[\frac{\mathrm{m}}{\mathrm{s}}\right]$,

$\sigma$-standard deviation $\left[\frac{m}{s}\right]$.

\section{Results}

The 18 series of measurements were collected:

- series \#1 - \#3 - with photodiode offset $V_{\text {offset }}=1 \mathrm{~V}$; with separation transformers not included (without separation from the building electrical grid noise) (see tab. 2),

- series \#4-\#10 - with photodiode offset $V_{\text {offset }}=4 \mathrm{~V}$; with separation transformers not included (see tab. 3),

- series \#11 - \#18 - with photodiode offset $V_{\text {offset }}=4 \mathrm{~V}$, with separation transformers included (with separation from the building electrical grid noise) (see tab. 4).

The measurements were repeated of 30 times per series.

We have started the investigation of the precision under repeatability conditions of measurements, with the standard offset photodiode voltage $V_{\text {offset }}=1 \mathrm{~V}$. During three series (\#1 - \#3) for a different angle of the incident light beam $(\theta)$, quite high standard deviations $\left(3 \sigma^{\prime} \mathrm{s}\right)$ for linear speed measurements were discovered, together with unacceptable low capability factors $C_{g}$ 's (see tab. 2).

Table 2. The very first experimental results (series \#1 - \#3) for the photodiode offset voltage $V_{\text {offset }}=$ $1 \mathrm{~V}$

\begin{tabular}{ccccccc}
\hline Series \# & $V_{\text {offset }}[\mathrm{V}]$ & $\theta\left[^{\circ}\right]$ & $R[\mathrm{~mm}]$ & $\bar{v}[\mathrm{~m} / \mathrm{s}]$ & $3 \sigma[\mathrm{m} / \mathrm{s}]$ & $C_{g}$ \\
\hline$\# 1$ & 1 & 88.50 & 30 & 5.51 & 0.45 & 0.18 \\
$\# 2$ & 1 & 78.75 & 30 & 5.41 & 0.39 & 0.21 \\
$\# 3$ & 1 & 67.50 & 30 & 5.52 & 0.27 & 0.31 \\
\hline
\end{tabular}

During the first three series, the setup was not capable at all for the precision laser Doppler velocimetry. The first step to improve it was to increase the offset voltage to $V_{\text {offset }}=4 \mathrm{~V}$ (see tab. 3). Higher $C_{g}{ }^{\prime} \mathrm{s}$ factors and lower $3 \sigma^{\prime}$ s) were appeared.

Table 3. The experimental results (series \#4 -\#10) for the photodiode offset voltage $V_{\text {offset }}=4 \mathrm{~V}$

\begin{tabular}{ccccccc}
\hline Series \# & $V_{\text {offset }}[\mathrm{V}]$ & $\theta\left[{ }^{\circ}\right]$ & $R[\mathrm{~mm}]$ & $\bar{v}[\mathrm{~m} / \mathrm{s}]$ & $3 \sigma[\mathrm{m} / \mathrm{s}]$ & $C_{g}$ \\
\hline$\# 4$ & 4 & 88.50 & 30 & 5.49 & 0.15 & 0.54 \\
$\# 5$ & 4 & 78.75 & 30 & 5.46 & 0.12 & 0.72 \\
$\# 6$ & 4 & 67.50 & 30 & 5.52 & 0.21 & 0.40 \\
$\# 7$ & 4 & 78.75 & 25 & 4.43 & 0.21 & 0.33 \\
$\# 8$ & 4 & 67.50 & 25 & 4.58 & 0.12 & 0.53 \\
$\# 9$ & 4 & 78.75 & 20 & 3.57 & 0.12 & 0.46 \\
$\# 10$ & 4 & 67.50 & 20 & 3.61 & 0.09 & 0.57 \\
\hline
\end{tabular}

The important modification was, to use the electrical separation of the laboratory from the building electrical grid noise, by using the transformers (see sec. 2). Separation transformers are highly 
recommended for individual lab grounding electric circuit. A stable power supply in the lab, by using separation is essential, e.g. in highly sensitive quantum metrology [39-41], and we decided to do this as well. In this way, the noise signal was significantly reduced, and precision of the surface linear speed measurements improved (low $3 \sigma^{\prime}$ s) with an acceptable $C_{g}$ 's factors (see tab. 4 ).

Table 4. The experimental results (series \#11 - \#18) with setup connected through the isolation transformers $\left(V_{\text {offset }}=4 \mathrm{~V}\right)$

\begin{tabular}{ccccccc}
\hline Series \# & $V_{\text {offset }}[\mathrm{V}]$ & $\theta\left[^{\circ}\right]$ & $R[\mathrm{~mm}]$ & $\bar{v}[\mathrm{~m} / \mathrm{s}]$ & $3 \sigma[\mathrm{m} / \mathrm{s}]$ & $C_{g}$ \\
\hline$\# 11$ & 4 & 78.75 & 30 & 5.54 & 0.12 & 0.81 \\
$\# 12$ & 4 & 78.75 & 30 & 5.56 & 0.12 & 0.74 \\
$\# 13$ & 4 & 67.50 & 30 & 5.57 & 0.09 & 1.07 \\
$\# 14$ & 4 & 67.50 & 30 & 4.48 & 0.09 & 1.21 \\
$\# 15$ & 4 & 78.75 & 25 & 4.24 & 0.09 & 1.00 \\
$\# 16$ & 4 & 78.75 & 25 & 4.22 & 0.09 & 0.71 \\
$\# 17$ & 4 & 67.50 & 25 & 4.25 & 0.06 & 1.00 \\
$\# 18$ & 4 & 67.50 & 25 & 4.26 & 0.09 & 0.83 \\
\hline
\end{tabular}

By improving the signal with $V_{\text {offset }}$ adjusting and especially, by electric noise reduction with separation using the transformers, the $C_{g}$ 's factors were found in the correct level $\sim 1$, which means, the measurement system was capable enough for this purpose. In this way, we also proved, to achieve high precision, under repeatability conditions, of the surface linear speed measurement, some part of the information-rich metrology paradigm is good to be fulfilled [42]. It is important as well as for surface texture measurements. For frequency measurements, the electrical grid of the laboratory should be separated from the building grid noise.

The temperature in the laboratory during the measurements $\left(20 \pm 1\left[{ }^{\circ} \mathrm{C}\right]\right)$ has been monitored. The mechanical and acoustic noises were reduced by the setup construction. Other disturbing effects in the experiment, e.g. humidity, were negligible when the optical fibre setup is used.

All measurement results of the disc speed were normally distributed (see e.g. tab. 5 and fig. 16-19), it means, standard deviation parameters ( $\sigma^{\prime}$ s, in this proposal $3 \sigma^{\prime}$ s) are related to the measurements uncertainties $[43,44]$. In fig. 17 and 19 examples of the quantile-quantile plots are presented. These probability plots are helpful to check if the normality assumption is plausible, and if not, how the assumption is violated and what data points contribute to the violation [45]. The normality is easy to see along the straight line, with the points in the confidence bands area [46].

As an extra check for the normality of the data distribution, the Shapiro-Wilk test was used (see e.g. tab. 5).

Table 5. Shapiro-Wilk normality test results for series \#1 and \#15 (W - is the statistic used in the hypothesis testing [36], p-value - is the probability value)

\begin{tabular}{ccc}
\hline Series \# & W & p-value \\
\hline$\# 1$ & 0.99 & 0.98 \\
$\# 15$ & 0.98 & 0.77 \\
\hline
\end{tabular}




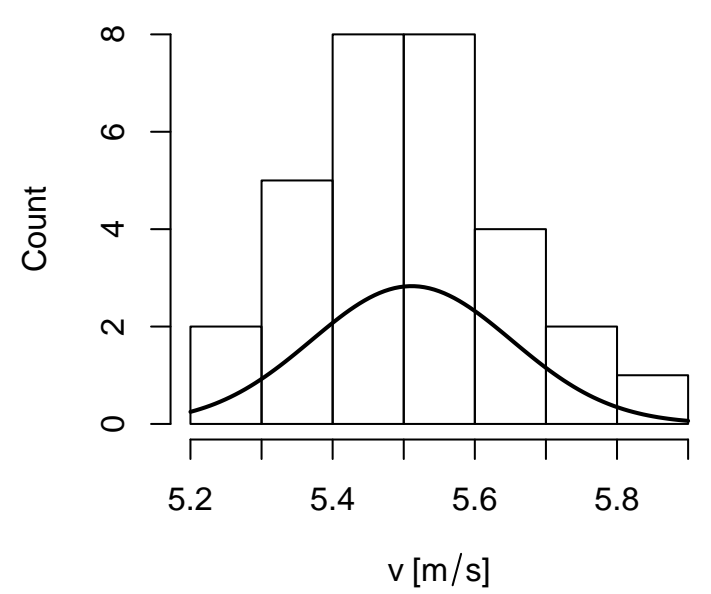

Figure 16. The normal distribution of the data in series \#1: $I=25 \mathrm{~mA}$, $V_{\text {offset }}=1 \mathrm{~V}, \theta=88.5^{\circ}, R=30 \mathrm{~mm}$, $\bar{v}=5.51[\mathrm{~m} / \mathrm{s}], \sigma=0.15[\mathrm{~m} / \mathrm{s}]$

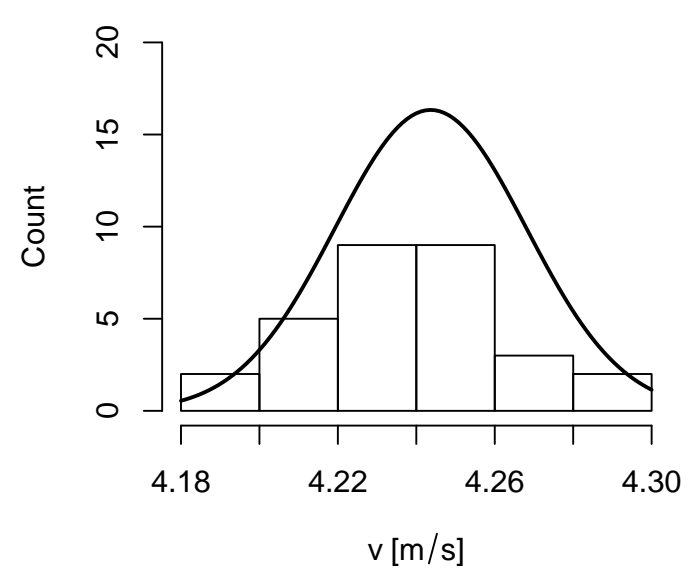

Figure 18. The normal distribution of the data in series \#15: $I=25 \mathrm{~mA}$, $V_{\text {offset }}=4 \mathrm{~V}, \theta=78.75^{\circ}, R=25 \mathrm{~mm}$, $\bar{v}=4.24[\mathrm{~m} / \mathrm{s}], \sigma=0.03[\mathrm{~m} / \mathrm{s}]$

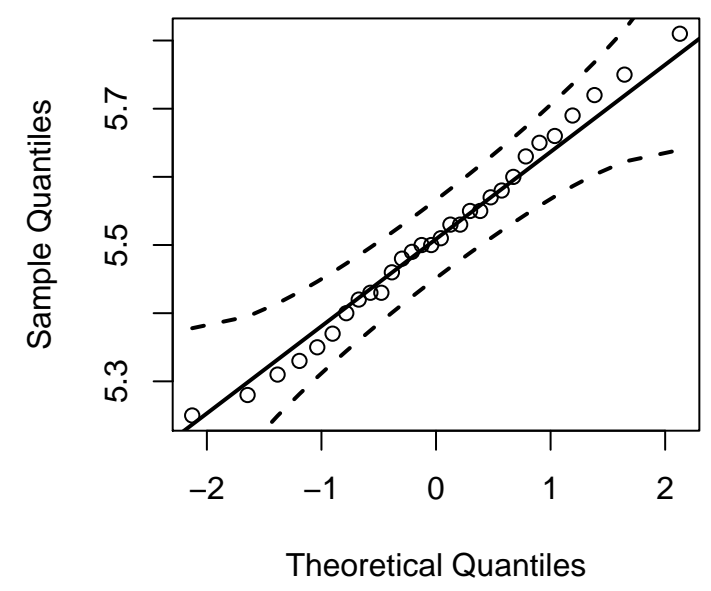

Figure 17. The Q-Q plot for data in series \#1: $I=25 \mathrm{~mA}, V_{\text {offset }}=1 \mathrm{~V}$, $\theta=88.5^{\circ}, R=30 \mathrm{~mm}, \bar{v}=5.51[\mathrm{~m} / \mathrm{s}]$, $\sigma=0.15[\mathrm{~m} / \mathrm{s}] . \quad(1-\alpha=0.95)$ (Theoretical quantiles are the quantiles from the standard Normal distribution with mean 0 and standard deviation 1 ; $\alpha$ is the significance level 0.05 )

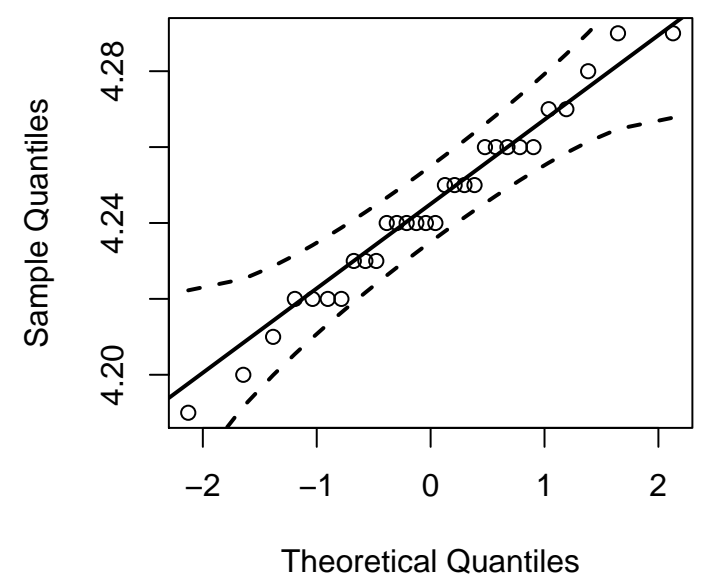

Figure 19. The Q-Q plot for data in series \#15: $I=25 \mathrm{~mA}, V_{\text {offset }}=$ $4 \mathrm{~V}, \theta=78.75^{\circ}, R=25 \mathrm{~mm}, \bar{v}=$ $4.24[\mathrm{~m} / \mathrm{s}], \sigma=0.03[\mathrm{~m} / \mathrm{s}] .(1-\alpha=0.95)$ (Theoretical quantiles are the quantiles from the standard Normal distribution with mean 0 and standard deviation 1 ; $\alpha$ is the significance level 0.05 )

The disc speed (as average $\bar{v}$ ) versus disc radius $(R)$ graph for measured values (see fig. 20) shows full correspondence to the linear relation of the $v=f(R)$ (see eq. 6 and compare fig. 20 with fig. 14). The disc speed (as average $\vec{v}$ ) versus angle $\theta-\Varangle(\vec{v}, \vec{c})$ graph for measured values shows correctly a constant line (see fig. 21). The slope of the line is related to the measurement errors evaluated. 


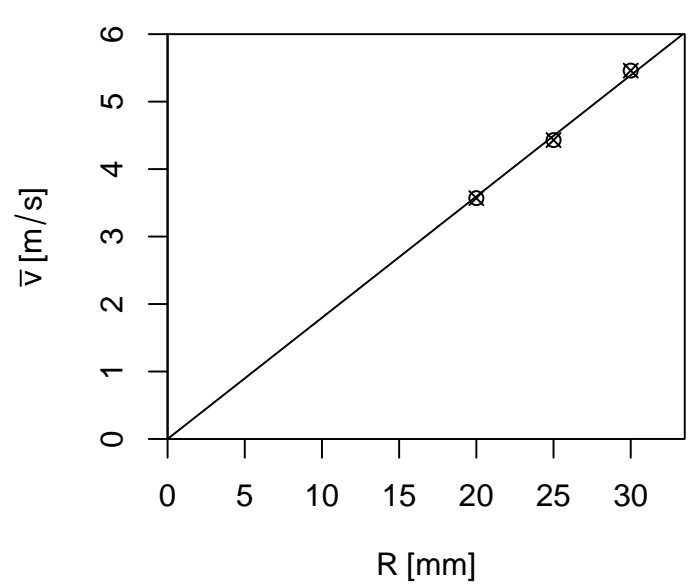

Figure 20. The $\bar{v}=f(R)$ plot for series $\# 4$,\#7, \#9 from tab. 3

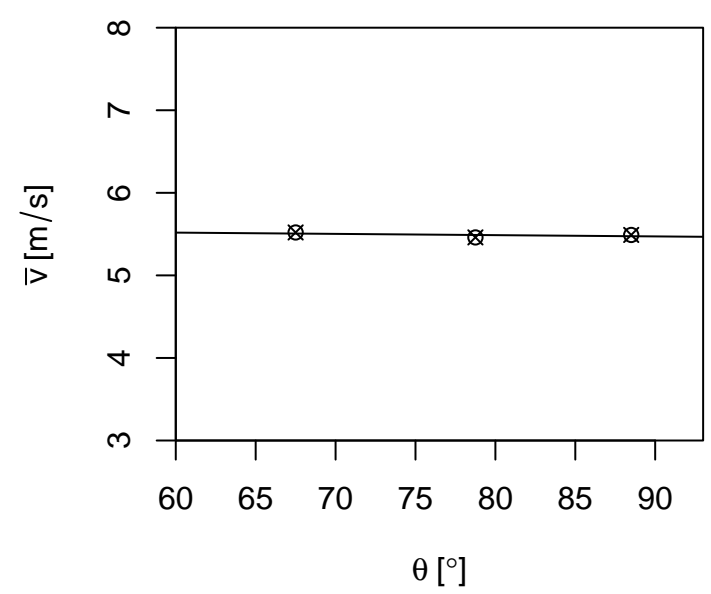

Figure 21. The $\bar{v}=f(\theta)$ plot for series $\# 4, \# 5$, \#6 from tab. 3

\section{Discussion}

In the Industry 4.0 age, significant progress in surface metrology has been observed. Optical metrology is going to be a game-changer in that field. More research is needed to simplify the hardware and software of the systems, with well known and controlled electric parameters of the systems power supply, as well.

In the paper, a simple modification of the Doppler kit is presented. The laboratory electric grid separation from the building grid noise, and self-built DC engine stable power supply, improved fairly enough the setup to be used as the low-cost, high precision measurement system. For a fast surface Doppler-shift velocimetry, the precision under repeatability conditions of measurements has been evaluated. The precision of the linear speed measurements with the setup was above $0.2 \frac{\mathrm{m}}{\mathrm{s}}$. It was estimated as the standard deviations $\left(3 \sigma^{\prime} \mathrm{s}<0.2\right)$, based on the normal distribution of the data. The capability factors $C_{q}$ 's, in final experiments, were in a close range of $\sim 1$, which means, the setup was capable enough for this metrological application, and modifications presented were essential.

Here we postulate; not only the temperature, pressure, humidity or vibrations stabilisation/isolation are essential for high precision measurements, but the power supply stability of the system as well. Or even more, in fast, short-time measurements, where others effects are not disturbing so much. The electric circuit conditions of the power supply should be well known before the measurements and kept controlled well.

Further investigations with various surfaces and measurement instruments will begin presently. The surface reflectivity, polarisation state of light, different object displacement, needs to be further investigated. The proposed setup is low-cost, fast, customisable, and by using optical fibres can be used for surface speed measurements, with a long-distance between the object and the unit. Using the optical fibres might be an advantage for real-time online measurements, where standard optical systems, can not be used due to the environmental conditions.

The rise of new metrology tools has seen suppliers focus their attention only on the hardware devices, with everyone focusing mainly on speed, accuracy, portability. However, the one thing that makes all of these devices work and produce measurement results is the software. It seems that manufacturers seem to have created their own software that works only with their products. By accepting a manufacturer's software, users are immediately limited to what that brand can offer in the future.

In the article, the hardware modification is presented, but also the data evaluation as well. By using the open-source $\mathrm{R}$ programming language, the signal processing and statistics were given. With the open-source codes, the mathematics behind the data analysis steps are open to the public and can be inspected or even corrected by others. Some ongoing projects on the metrology background are quite promising [47]. 
After more improvements, the low-cost modified Doppler kit might be used in a complex system as a part responsible for precision surface displacement measurements [27].

\section{Conclusion}

A simple modification of the training Doppler kit by using separation transformers and self-built power supply has been presented. Signal improvements and data evaluation have been shown. An improvement of measurement precision using statistic was evaluated. For signal processing and data analysis, an open-source R programming language was proposed. Based on the well known Doppler radar technique, the proposed setup is promising as a low-cost, simple, fast, alternative system for high precision surface velocimetry.

Author Contributions: Conceptualization, Dawid Kucharski; Data curation, Dawid Kucharski and Mateusz Musiałowski; Formal analysis, Dawid Kucharski; Investigation, Dawid Kucharski and Mateusz Musiałowski; Project administration, Dawid Kucharski; Software, Dawid Kucharski; Supervision, Dawid Kucharski; Writing original draft, Dawid Kucharski; Writing - review \& editing, Dawid Kucharski.

All authors have read and agreed to the published version of the manuscript.

Funding: This research was funded by Poznan University of Technology grant number: 02/22/SBAD/1501.

Conflicts of Interest: The authors declare no conflict of interest. The funder had no role in the design of the study; in the collection, analyses, or interpretation of data; in the writing of the manuscript, or in the decision to publish the results.

\section{References}

1. The International Bureau of Weights and Measures. The International System of Units (SI). Technical Report 9, 2019.

2. Stenger, J.; Ullrich, J. Units Based on Constants: The Redefinition of the International System of Units. Annu. Rev. Condens. Matter Phys. 2016, 7, 35-59. doi:10.1146/annurev-conmatphys-031115-011311.

3. Leach, R. Optical Measurement of Surface Topography; Springer Berlin Heidelberg: Berlin, Heidelberg, 2011; p. 323. doi:10.1007/978-3-642-12012-1.

4. Pawlus, P.; Reizer, R.; Wieczorowski, M. Comparison of results of surface texture measurement obtained with stylus methods and optical methods. Metrol. Meas. Syst. 2018, vol. 25, 589-602. doi:10.24425/123894.

5. Leach, R.; Bourell, D.; Carmignato, S.; Donmez, A.; Senin, N.; Dewulf, W. Geometrical metrology for metal additive manufacturing. CIRP Ann. 2019, 68, 677-700. doi:10.1016/j.cirp.2019.05.004.

6. Gapiński, B.; Wieczorowski, M.; Marciniak-Podsadna, L.; Swojak, N.; Mendak, M.; Kucharski, D.; Szelewski, M.; Krawczyk, A. Use of White Light and Laser 3D Scanners for Measurement of Mesoscale Surface Asperities. In Lect. Notes Mech. Eng.; 2019; pp. 239-256. doi:10.1007/978-3-030-18682-1_19.

7. Gao, W.; Haitjema, H.; Fang, F.; Leach, R.; Cheung, C.; Savio, E.; Linares, J. On-machine and in-process surface metrology for precision manufacturing. CIRP Ann. 2019, 68, 843-866. doi:10.1016/j.cirp.2019.05.005.

8. Xue, B.; Zhang, H.; Zhao, T.; Jing, H. A Traceable High-Accuracy Velocity Measurement by Electro-Optic Dual-Comb Interferometry. Appl. Sci. 2019, 9, 4118. doi:10.3390/app9194118.

9. Doppler, C. Über das farbige Licht der Dopplersterne und einiger anderer Gestirne des Himmels. Abhandlungen der Königlichen Böhmischen Gesellschaft der Wissenschaften1 1842. doi:10.1007/s13398-014-0173-7.2.

10. McMillan, C.F.; Goosman, D.R.; Parker, N.L.; Steinmetz, L.L.; Chau, H.H.; Huen, T.; Whipkey, R.K.; Perry, S.J. Velocimetry of fast surfaces using Fabry-Perot interferometry. Rev. Sci. Instrum. 1988, 59, 1-21. doi:10.1063/1.1140014.

11. Jaroszewicz, L.R.; Krajewski, Z. Optical fibre interferometric system for Doppler effect measurement. Opto-electronics Rev. 2002, 10, 185-191.

12. Tatam, R.P. Planar Doppler velocimetry using optical fibers. Interferom. XII Appl.; Osten, W.; Novak, E., Eds., 2004, Vol. 5532, p. 50. doi:10.1117/12.562140.

13. Charrett, T.O.H.; James, S.W.; Tatam, R.P. Optical fibre laser velocimetry: a review. Meas. Sci. Technol. 2012, 23, 032001. doi:10.1088/0957-0233/23/3/032001. 
14. Hu, Y.; Miyashita, L.; Watanabe, Y.; Ishikawa, M. Visual Calibration for Multiview Laser Doppler Speed Sensing. Sensors 2019, 19, 582. doi:10.3390/s19030582.

15. Berkovic, G.; Shafir, E. Optical methods for distance and displacement measurements. Adv. Opt. Photonics 2012, 4, 441. doi:10.1364/AOP.4.000441.

16. Meijer, F.; Kucharski, D.; Stachowska, E. Determination of the phase in the center of a circular two-beam interference pattern to determine the displacement of a rough surface. Opt. Eng. 2018, 57, 1. doi:10.1117/1.OE.57.10.104101.

17. Nieh, J.Y.; Cheng, Y.P. Innovative Multi-Target Estimating with Clutter-Suppression Technique for Pulsed Radar Systems. Sensors 2020, 20, 2446. doi:10.3390/s20092446.

18. Hyun, E.; Jin, Y. Doppler-spectrum feature-based human-vehicle classification scheme using machine learning for an FMCW radar sensor. Sensors (Switzerland) 2020, 20. doi:10.3390/s20072001.

19. Malešević, N.; Petrović, V.; Belić, M.; Antfolk, C.; Mihajlović, V.; Janković, M. Contactless Real-Time Heartbeat Detection via $24 \mathrm{GHz}$ Continuous-Wave Doppler Radar Using Artificial Neural Networks. Sensors 2020, 20, 2351. doi:10.3390/s20082351.

20. Gouveia, C.; Vieira, J.; Pinho, P. A review on methods for random motion detection and compensation in bio-radar systems. Sensors (Switzerland) 2019, 19, 1-17. doi:10.3390/s19030604.

21. Einstein, A. Zur Elektrodynamik bewegter Körper. Ann. Phys. 1905, 322, 891-921. doi:10.1002/andp.19053221004.

22. Truax, B.E.; Demarest, F.C.; Sommargren, G.E. Laser Doppler velocimeter for velocity and length measurements of moving surfaces. Appl. Opt. 1984, 23, 67. doi:10.1364/AO.23.000067.

23. Matsubara, K.; Stork, W.; Wagner, A.; Drescher, J.; Müller-Glaser, K.D. Simultaneous measurement of the velocity and the displacement of the moving rough surface by a laser Doppler velocimeter. Appl. Opt. 1997, 36, 4516. doi:10.1364/AO.36.004516.

24. Moir, C.I. Miniature laser doppler velocimetry systems. Opt. Sensors 2009; Baldini, F.; Homola, J.; Lieberman, R.A., Eds., 2009, Vol. 7356, p. 73560I. doi:10.1117/12.819324.

25. Idil Fibres Optiques. Fibre Optic Interferometry and Doppler - User Guide, 1.3 ed.; pp. 160-161.

26. Safibra. Fiber optic interferometry training kit, 2020.

27. Kucharski, D.; Zdunek, H. A low-cost, simple optical setup for a fast scatterometry surface roughness measurements with nanometric precision. Bull. Polish Acad. Sci. Tech. Sci. 2020, 68, 485-490. doi:10.24425/bpasts.2020.133364.

28. Roussel, J. FEMTO - Cours d'optique, 2015.

29. Roussel, J. Figures TIKZ du cours "L'effet Doppler", 2020.

30. Seilmayer, M. spectral: Common Methods of Spectral Data Analysis, 2019.

31. R Core Team. R: A Language and Environment for Statistical Computing. R Foundation for Statistical Computing, Vienna, Austria, 2020.

32. Montgomery, D.C. Introduction To Statical Quality Control, 6th ed.; John Wiley \& Sons, Inc., 2009; pp. 344-390.

33. Polák, P.; Drlička, R.; Žitňanský, J. Capability assessment of measuring equipment using statistic method. Manag. Syst. Prod. Eng. 2014, 4, 184-186. doi:10.12914/MSPE-08-04-2014.

34. Kucharski, D.; Nowak, J. A simple setup for repeatability analysis of a low-level laser therapy scanner. In Adv. Intell. Syst. Comput.; Gzik, M.; Tkacz, E.; Paszenda, Z.; Piętka, E., Eds.; Springer International Publishing: Cham, 2018; Vol. 623, Advances in Intelligent Systems and Computing, pp. 97-105. doi:10.1007/978-3-319-70063-2_11.

35. Kucharski, D.; Michalska, M. Repeatability Investigations of a Handheld Electronic Spirometer. In Innov. Biomed. Eng.; Gzik, M.; Tkacz, E.; Paszenda, Z.; Piętka, E., Eds.; Springer International Publishing: Cham, 2019; pp. 77-84. doi:10.1007/978-3-030-15472-1_9.

36. Shapiro, S.S.; Wilk, M.B.; Chen, H.J. A Comparative Study of Various Tests for Normality. J. Am. Stat. Assoc. 1968, 63, 1343-1372. doi:10.1080/01621459.1968.10480932.

37. Ihaka, R.; Gentleman, R. R: A Language for Data Analysis and Graphics. J. Comput. Graph. Stat. 1996, 5, 299-314. doi:10.1080/10618600.1996.10474713.

38. Tippmann, S. Programming tools: Adventures with R. Nature 2015, 517, 109-110. doi:10.1038/517109a.

39. Charles Doret, S.; Amini, J.M.; Wright, K.; Volin, C.; Killian, T.; Ozakin, A.; Denison, D.; Hayden, H.; Pai, C.S.; Slusher, R.E.; Harter, A.W. Controlling trapping potentials and stray electric fields in a 
microfabricated ion trap through design and compensation. New J. Phys. 2012, 14, 073012, [1204.4147]. doi:10.1088/1367-2630/14/7/073012.

40. Kumph, M.; Holz, P.; Langer, K.; Meraner, M.; Niedermayr, M.; Brownnutt, M.; Blatt, R. Operation of a planar-electrode ion-trap array with adjustable RF electrodes. New J. Phys. 2016, 18, 023047, [1402.0791]. doi:10.1088/1367-2630/18/2/023047.

41. Lakhmanskiy, K.; Holz, P.C.; Schärtl, D.; Ames, B.; Assouly, R.; Monz, T.; Colombe, Y.; Blatt, R. Observation of superconductivity and surface noise using a single trapped ion as a field probe. Phys. Rev. A 2018, 99, 023405, [1805.00793]. doi:10.1103/PhysRevA.99.023405.

42. Senin, N.; Leach, R. Information-rich surface metrology. Procedia CIRP 2018, 75, $19-26$. doi:10.1016/j.procir.2018.05.003.

43. Bell, S. Measurement good practice guide No. 11. Natl. Phys. Lab. 2004, pp. 1-41.

44. Dietrich, E.; Schulze, A. Statistical Procedures for Machine and Process Qualification; Hanser, 2010.

45. Wickham, H. ggplot2: Elegant Graphics for Data Analysis; Springer-Verlag New York, 2016.

46. Almeida, A.; Loy, A.; Hofmann, H. qqplotr: Quantile-Quantile Plot Extensions for 'ggplot2', 2017.

47. Ellison., S.L.R. metRology: Support for Metrological Applications, 2018. 